\title{
HYBRID SIMULATION FOR MODELLING LARGE SYSTEMS: AN EXAMPLE OF INTEGRATED CARE MODEL
}

\author{
Jafri Zulkepli \\ Tillal Eldabi \\ Brunel Business School \\ Brunel University \\ Uxbridge UB8 3PH, Middlesex, UK
}

\author{
Navonil Mustafee \\ Swansea University \\ College of Business, Economics and Law \\ Swansea SA2 8PP, Wales, UK
}

\begin{abstract}
Developing models for large systems is not a trivial task. Using only Discrete Event Simulation (DES) as a modelling technique may mean that the complexity of the underlying model will increase exponentially with the size of the model. An alternative to this is the use of System Dynamics (SD) for modeling large systems using the positive and negative feedback loops. However, for modelling a human-centric system like healthcare, DES is important as it provides individuality analysis; similarly, SD is important as it facilitates the whole systems approach. The combined application of OR/Simulation methods enable the symbiotic realization of the strengths of individual techniques, while reducing their limitations; in this paper it is suggested that a combined SD-DES approach (also referred to as hybrid technique) can be effectively used for modelling large systems. The example being used in this context is the modelling of an Integrated Care (IC) system in healthcare.
\end{abstract}

\section{INTRODUCTION}

Simulation methods enable stakeholders to analyze and evaluate strategies for effective management of complex systems. It is therefore not surprising that an increasing number of studies have used a plethora of Operations Research/Simulation methods, such as Optimization, Markov Chains, Linear Programming, DES, SD, Forecasting, Just-In-Time, Decision Trees and Soft Systems Analysis, to facilitate better and more informed decision making. These techniques have been frequently applied in isolation. However, the complexity of systems being modeled and their multi-faceted relationships may mean that combining OR/Simulation techniques will reduce the limitations and increase the capabilities of the individual methods, thereby potentially realizing synergies across techniques and facilitating greater insights to problem solving (Fakhimi and Mustafee 2011); it has also been argued that combined methods could aid stakeholder acceptance (Sachdeva, Williams, and Quigley 2007).

In this paper the combined application of OR/Simulation techniques will be referred to as a hybrid technique. The hybrid techniques in OR/Simulation have been applied in various domains, such as, construction, e.g., combined discrete-event and continuous simulation (AbouRizk and Wales 1997); container loading, e.g., combined application of Agent-Based Simulation and load plan construction heuristics (Mustafee and Bischoff 2011; Mustafee et al. 2011); healthcare, e.g., combined application of SD and DES (Chahal and Eldabi 2008), combined DES and ant colony optimization (Brailsford et al. 2007); modeling service and manufacturing activities pertaining to supply chains, e.g., combined application of analytic hierarchy process technique, SD and DES (Rabelo et al. 2007). The focus of this paper is the combined application of OR/Simulation techniques for modeling large systems in the healthcare context. The specific example is that of modeling integrated care system through a combination of SD and DES. 
The remainder of the paper is structured as follows. In section 2 we present an overview of literature on the application of multiple OR/Simulation technique within the UK healthcare context. Following this, in section 3 we describe the SD-DES approach to hybrid simulation. The integrated care system example is presented in section 4 whilst section 5 presents the interpretation of the results. Section 6 is the concluding section of the paper; it discusses some of the challenges of hybrid SD-DES modelling and draws the paper to a close.

\section{APPLICATION OF MULTIPLE OR/SIMULATION TECHNIQUES IN HEALTHCARE}

The discussion presented in this section is based on the review conducted by Fakhimi and Mustafee (2012); the reader is referred to the aforementioned paper for further information on the literature review methodology that was employed for the purposes of conducting this review. It is also to be noted here that unlike several other studies that have profiled literature in healthcare (e.g., Katsaliaki and Mustafee 2012; Brailsford et al. 2009; Jun, Jacobson, and Swisher 1999), the scope of the aforementioned review was not limited to simulation but it encompassed the overarching subject area of OR.

From a total of 70 healthcare papers that were included in the review, 26 studies $(37 \%)$ employed Multiple OR/Simulation Techniques (MORT) for the realization of their stated objectives. However, this number was higher when compared to the application of any one particular OR/Simulation technique for example, there were only 18 studies that reported the use of DES, the use of Monte-Carlo Simulation was reported in 11 papers, Mathematical Modeling (10 papers), Cohort Simulation (1), Statistical Quality Control Techniques (1), Scheduling (1), etc. The review also found that $80 \%$ of the papers (56 studies) included the application of simulation (DES, SD, ABS, etc.) either on its own, or together with a complimentary OR/Simulation technique.

The survey has identified the application of multiple techniques in relation to the evaluation of economic efficiency and cost-effectiveness of new forms of treatment, and to analyze costs and benefits related to social health issues such as infection and communicable disease. The majority of the studies have used Monte-Carlo Simulation together with Markov Chains, for example, to evaluate the costs and benefits of drugs used for the prevention of cardiac events, to estimate the cost effectiveness of different therapies for Alzheimer; to compare the cost-utility of two kinds of treatment for atopic eczema, and for comparing the costs and benefits of alternate treatments for type 2 diabetes patients. One study has applied decision analytical modeling technique and Markov framework to evaluate the cost and clinical effectiveness of chemotherapy drug for breast cancer patients. Next, DES and Markov modeling has been used in the context of comparative evaluation of CRT clinical and cost-effectiveness for patients with heart failure. Another example of the application of MORT is the use of Forecasting and Length of Stay (LOS) modeling for the cost-effective analysis and modeling of occupancy time in the NHS.

Although the review identified several DES studies conducted in relation to the National Health Service in the UK (the search terms used in ISI Web of Knowledge $e^{S M}$ included ["NHS" OR "National Health Service" OR "UK" OR "England" OR "Wales" OR "Scotland" OR "Northern Ireland"] in any of the following fields - title, abstract, authors' keywords and keywords plus), there were no papers that reported the use of hybrid technique using SD and DES (however, it is to be noted that there are several conference papers on this particular topic, some of which are included in the reference list). It can therefore be argued that further research is required for the application of combined SD-DES modelling for healthcare problems (including development of methodology and associated frameworks), together with exemplar applications of the combined approach to real world healthcare problems, and dissemination of this work in impact factor journals.

\section{COMBINED SD-DES SIMULATION - AN EXAMPLE OF HYBRID TECHNIQUE}

SD and DES are two simulation techniques that have been applied in a hybrid context in software engineering, manufacturing, etc. Combining these two techniques provides huge advantages to the model and thus improves the decision making process. Brailsford (2008) suggested that although combining DES 
and SD will be challenging, it will be beneficial especially in the healthcare sector since the DES component will permit detailed, individual patient analysis and SD will facilitate the whole systems approach. Most researchers have argued that although these simulation branches have a common motivation but there have been limited efforts to combine them (Sweester 1999, Lane 2000, Brailsford and Hilton, 2001, Moorcroft and Robinson, 2005). By implementing and applying hybrid techniques, it is argued that the decision making process will be improved and enhanced. In this section we specifically focus on hybrid modeling for large systems.

Developing models for large systems such as an integrated care system is complicated. The system comprises of many stakeholders (clinicians, policy makers, NHS managers, end users, etc.) and the underlying processes involve the handling of patients as well as care pathways between different healthcare service providers (e.g., critical care, intermediate care and social care). Developing large systems' models will need sufficient budget allocation and will also require significant investments in human resources and time. There are several examples in literature related to the modeling of Integrated Care (IC) systems using either SD or DES, for example, Desai et al. (2008); Campbell, Karnon, and Dowie (2001); Katsaliaki et al. (2005); Wolstenhome et al. (2004). However, none of these studies have used a combined SD-DES hybrid technique to realize the study objectives, and the models that have been developed have used a single simulation technique - either SD or DES.

Developing an IC system using a single simulation technique will ignore some of the important features that are inherent in the healthcare domain. Using only DES to model the IC system will make the model loose the feedback loop feature that is provided by SD. Feedback loops are used in SD to model the cause and effect relationships (Sterman 2000). Although it is arguable that feedback loops can be modeled in DES (e.g., circular path between two workstations), the usage of this term in the paper is consistent with its definition in SD. The DES methodology is also prone to the development of more complex models since the complexity of an underlying DES model increases exponentially with the size of the model (Chahal and Eldabi 2008); this is also demonstrated in Mustafee et al. (2009). To solve this, some researchers are inclined to use SD since it affords itself to modeling large systems as a whole. However, as healthcare is a human-centric service and the patients have individual attributes, SD is not a complete solution since this modeling paradigm does not permit individual and detailed analysis of the constituent parts of a healthcare system (e.g., patients, clinician, and clinics). It is not practical to model individual elements (e.g., patients) using SD especially when the elements have different values (e.g., patient arrival time, patient Length Of Stay in particular wards); this is because SD models aggregate values (Brailsford and Hilton 2001). Therefore, it is suggested that combining SD and DES will enable the symbiotic realization of the strength of each technique, while reducing its limitations (Figure 1).

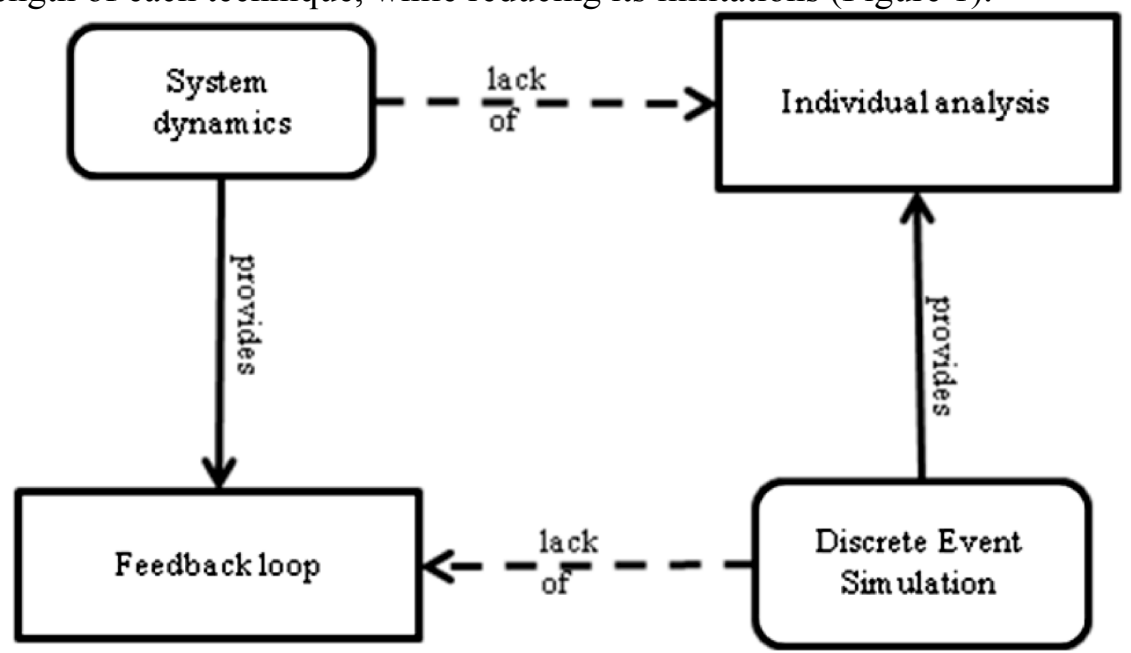

Figure 1: How Hybrid techniques can improve IC model 
Only a handful of studies have examined the applicability of hybrid SD-DES techniques for modeling systems; among them there are arguably only two studies that have presented a framework-based approach - Chahal, Eldabi, and Mandal (2009) and Helal et al. (2007). While Chahal, Eldabi and Mandal (2009) have applied their hybrid framework for modeling Accident \& Emergency Department, Helal et al. (2007) have applied their framework in the context of modeling a manufacturing supply chain. Although both of the aforementioned frameworks will guide the development of the integrated care model (section 4), it should be noted that neither of these frameworks specifically relate to the modeling of a large integrated care system.

\section{COMBINED SD-DES MODELLING OF AN INTEGRATED CARE (IC) SYSTEM}

\subsection{Linkages between IC, Intermediate Care and Social Care}

Several problems related to IC have been highlighted in literature, e.g., Mur-Veeman, van Raak, and Paulus (2008); Glendinning, Hudson, and Means (2005); van Raak, Paulus, and Mur-Veeman (2005); Bryan, Gage, and Gilbert (2005), and among them is the problem of late transfer to social care. This contributes to bed blocking since patients that are clinically ready to be transferred to social care continue to stay in the hospital until such time that a suitable place in social care can be allotted to them. In order to address this problem, intermediate care facilities have been introduced with the purpose of providing temporary placement to the patients while they await their transfer to social care and other forms of care management pathways. Important objectives that are realized through the creation of intermediate care facilities include, (a) they act as care facilities for the rehabilitation of the patients and serve as an intermediary between hospitals and social care; this is important considering that the older people are the main recipient of intermediate care and it prevents the transfer of frail older people patients to long-term care directly from an acute service (British Geriatrics Society 2008), (b) the problem of bed blocking is reduced and the resources in the hospitals can be released to those in need of acute treatment, (c) the care manger has longer time to assess the long-term care needs of the patients, (d) social care has more time to allocate care placements to the patients, (e) the healthcare team can ensure that patients are transferred to social care subsequent to their condition having improved; this prevents the readmission of patients, and (f) the intermediate care team helps the patients maintain their lives and provides a rehabilitation program and thus reduces their dependency on social care.

\subsection{The Hybrid IC Model}

The hybrid SD-DES modeling of IC consists of three models - a DES model for hospital care (we call it the Critical Care Module), a DES model for intermediate care (called the Assessment/Intermediate Care Module), and a SD model encompassing critical care, intermediate care and social care (we call this the IC module). Simul8 ${ }^{\mathrm{TM}}$ has been used for the DES models and Vensim ${ }^{\circledR}$ for SD modeling; the specific models are described next.

\subsubsection{The Critical Care Module (DES model)}

The Critical Care Module models the patient flow through a critical care facility, e.g., a hospital (Figure 2). It starts with the patient being admitted to the ward. The patient is then assessed by the doctor and will undergo some tests. Following the doctor's initial assessment some patients will be discharged, whereas others may require further assessment. A patient that needs to undergo a surgery procedure may be admitted to a surgical ward while waiting for the surgery. Subsequent to the operation the patient will be transferred to a recovery room for postoperative recovery. The patient will then be transferred to the normal ward and will remain here until medically fit to be discharged. There are two alternate postoperative pathways for the patient from this point on, (a) either the patient will be discharge, and (b) the patient will require continuing care services. The latter pathway is especially for the older peoples, and in this case the patient will be routed to the Assessment/Intermediate Care Module (section 4.2.2). 


\subsubsection{The Assessment/Intermediate Care Module (DES model)}

The Assessment/Intermediate Care Module incorporates the processes associated with the assessment of patients and it starts when patients that are medically fit and are ready for discharge. There will also be some patients that enter the system from outside the hospitals; these patients are referred to by other sources such as friends, relatives as well as GPs (Katsaliaki et al. 2005). The assessment begins with the patients' physical and psychological needs being gauged by occupational therapists and psychiatric nurses. This assessment ensures that the care placements to be allotted to the patients will meet their medical needs. Subsequent to this initial assessment, the care manager will assess whether the patients require personal care and whether they can carry out household tasks. This includes the assessment of the availability of formal/informal care as well as the provision of financial support (personal or public funds). Following this, a care package will be created and an appropriate care placement must be found. There is an additional condition where the care manager has to get a court order to act on behalf of the patient should the patient be invalid. The aforementioned assessments, which take-up a maximum of two weeks, are done while the patients are in intermediate care. Thus, the assessment and intermediate care processes are considered as one process and only one DES model is considered (Figure 3).

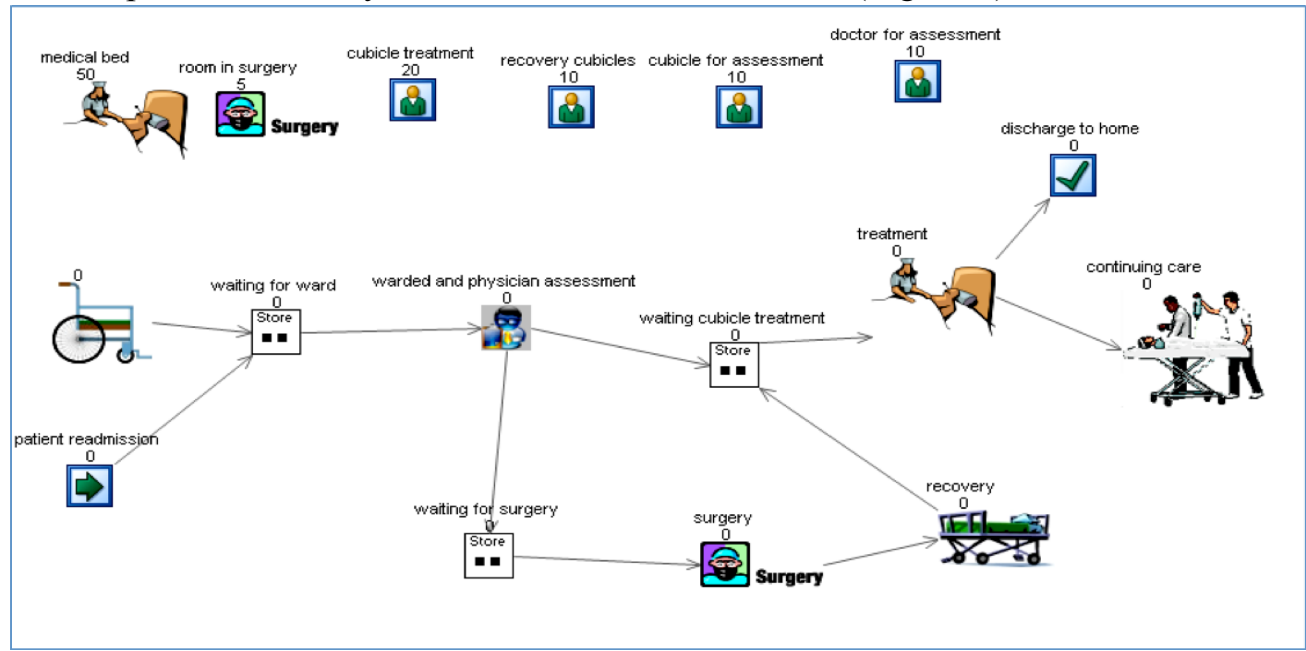

Figure 2: DES Model of the Critical Care Module

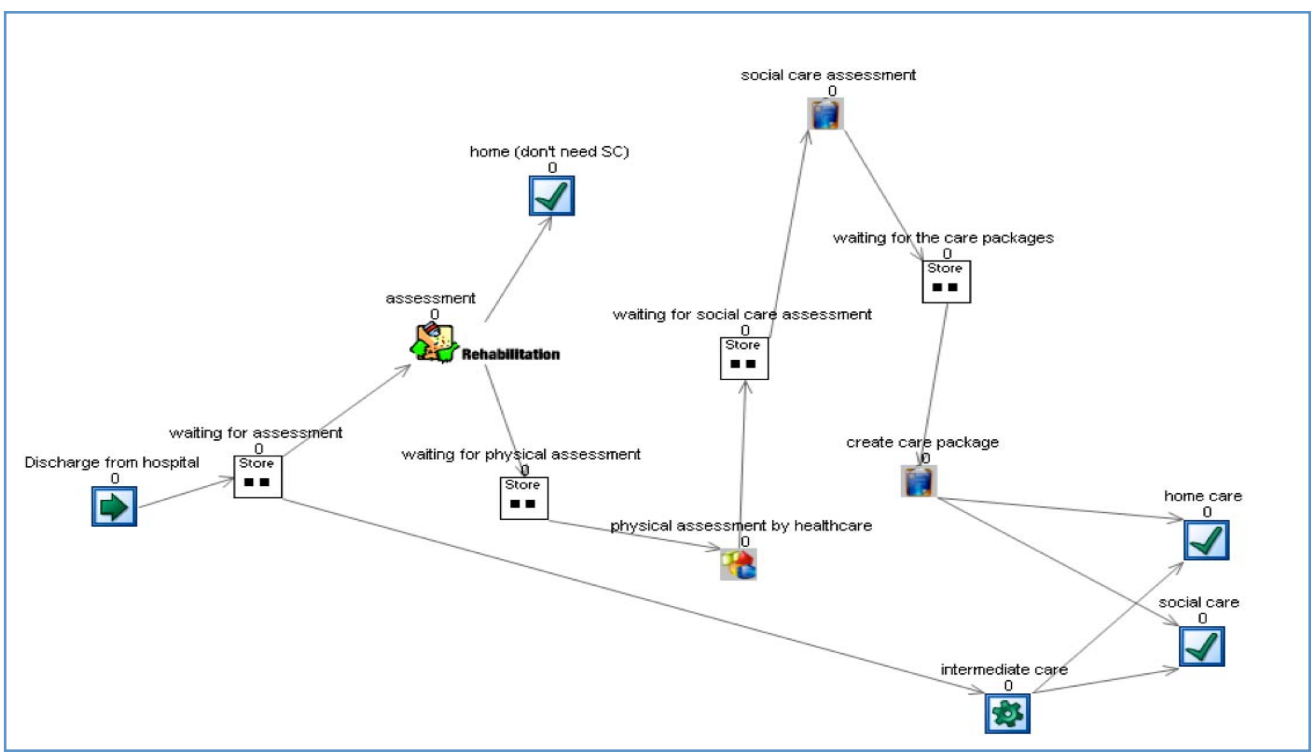

Figure3: DES Model of the Assessment/Intermediate Care Module 


\subsubsection{The IC Module (SD model)}

In our IC Module, SD is used for modeling intangible variables that relate to those providing care services to the patients (e.g., the nurses and the clinician). Examples of such variables include performance, motivation and stress level; these variables are influenced by the total number of patients entering the critical care, intermediate care and social care pathways. Figure 4 illustrates the SD model for the whole system of IC. Due to several limitations, including the lack of data and technical support, the authors only consider one type of output from the DES model that is used as an input (influencing factor) for the SD model. The DES output is the total number of patients entering acute care; this will, in turn, influence the stress level of the staff leading to increased patient discharge to the intermediate care. Whilst in intermediate care, the increase in patients numbers will add to the amount of time being spent on assessment, and this will also influence the stress level of staff leading to an increase in discharge rate and patient readmission (considering that due to the limitation of time and space, the patient are not ready for discharge, or not medically fit to be discharged). The acute care facilities have their own resources (beds) that will be occupied when patients are admitted to the hospital; these resources will be continued to be occupied until they are fully occupied. When this situation occurs, it will create bed blocking and will consequently increase the stress level of the clinicians. To make resources available again in critical care, it is arguable that the discharge rate to intermediate care will be increased. The same scenario may occur in intermediate care; Elf and Putilova (2005) argued that physical spaces will influence the patient's health and therefore, in order to create conducive places for healing, it is arguable that healthcare professionals may be forced to discharge some patients due to limited space availability.

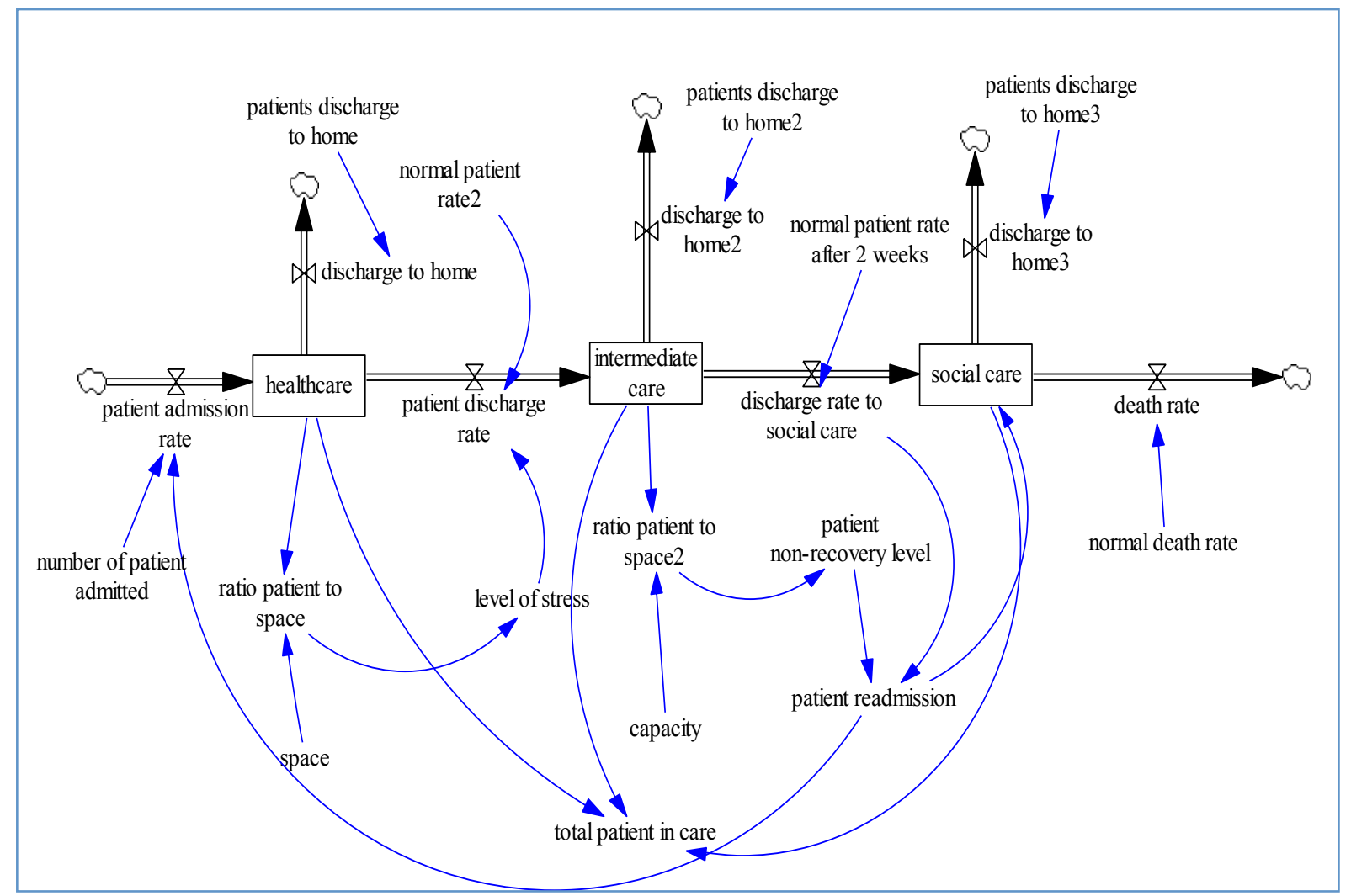

Figure 4: SD Model of the Integrated Care Module 


\subsubsection{Model Integration}

The hybrid SD-DES approach necessitates the identification of relationships among the variables present in DES and SD models. In the case of our IC model, the DES variable "number of patient admitted" from the Critical Care Module influences the SD variable "stress level" in the Integrated Care Module, wherein an increase in patient admission will result in an increase in the stress level among the healthcare professionals. As mentioned earlier, Elf and Putilova (2005) argued that physical spaces influence patients' health and therefore some patients may need to be discharged due to the lack of available spaces. This scenario might create another condition wherein patients may be readmitted to critical care, since, due to the stress level increase, the professional judment pertaining to patients' health may be wrong (i.e., the patient may not have been ready for discharge) and this may mean that the patient may have to be readmitted in the near future. Thus, we identify the realtionship between the following sets of variables:

- $\quad$ Patient admission $\rightarrow$ stress level $\rightarrow$ patient discharge rate

- $\quad$ Patient admission $\rightarrow$ stress level $\rightarrow$ patient readmission

The next step is to run the model and to experiment with interventions. The source DES model will be executed first. Subsequent to this the output from the source model will be collected and the resultant values will be used as inputs for the destination SD model. The SD model will then be executed. As patient readmission is the last output and is tangible, it will be inserted back into the DES model. The DES model will be executed again to provide new results after considering intangible factors, i.e. stress level and non-patient recovery level. The result of the combined SD-DES simulation should be considered as a whole in order to find the best result for implementation. It follows that all the variables from the source model that have been changed inorder to experiment with different interventions/policies should also be changed in the destination model. This is to ensure that the models are linked together and the results from both models are relaiable. Figure 5 shows how these variables are connected between models.

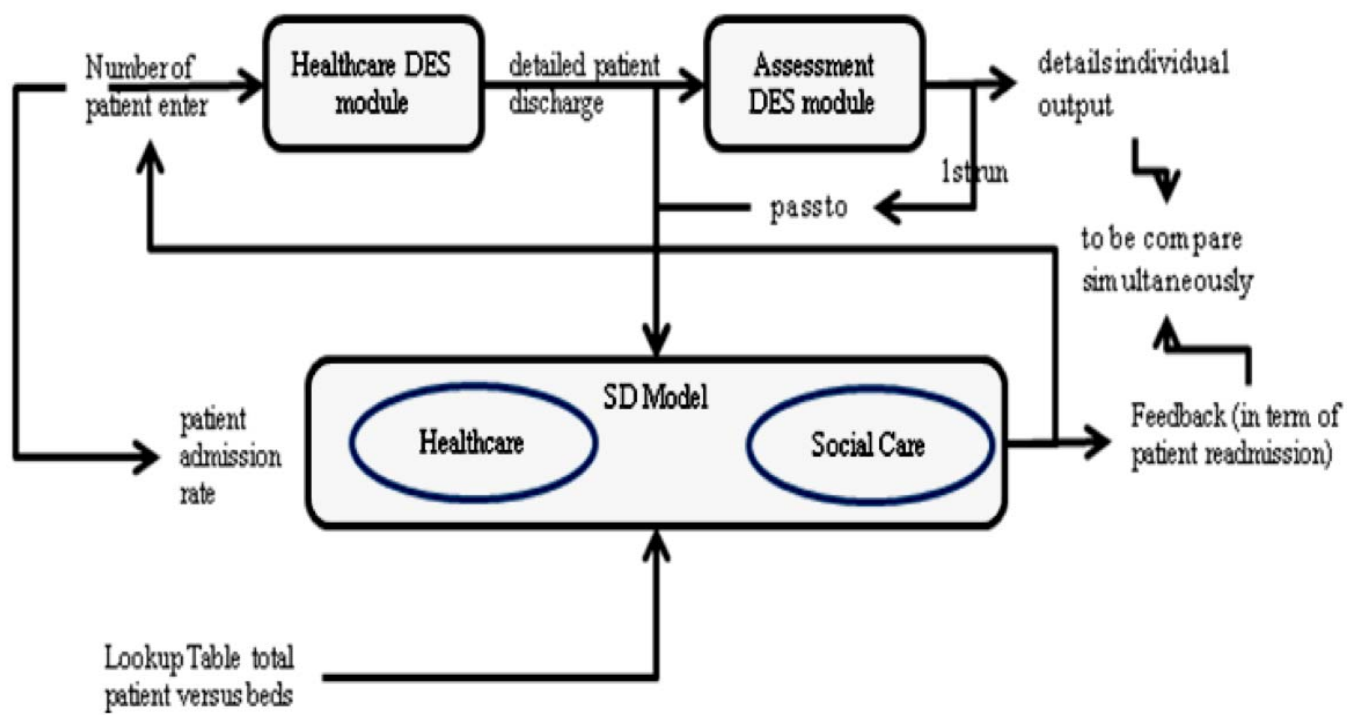

Figure 5: Models Communication

Model communcation is further explained in this paragraph. By simulating the DES model we are able to retrieve data pertaining to the total patient admission. This data is stored in an Excel file (unit of capture: weekly data). The Excel file provides input values to the SD model, and as the SD model executes it reads the total patient admission data from the file. We use 'lookup table' Excel function for the variable 'stress level' and use this to determine the patient discharge rate. As mentioned earlier, we 
assume that as the number of patients being admitted increases, the stress level of those attending to the patients also increases, and this, in turn, affects the rate of patient discharge. To obtain the total number of patient discharge, the stress level is multiplied with the number of total patients. A similar method is used for patient readmission.

\section{INTERPRETATION OF RESULTS}

The DES models will show output from the perspective of individual cases. Such outputs are total patient in waiting, waiting time and total patient admission to healthcare, social care as well as intermediate care. The SD model represents the output from the perspective of feedback which will be gathered from the level of stress and non-recovery patients. The feedback loop is total patients discharge from healthcare as a result of staffs' stress level and total patient readmission due to non-recovery patient. These feedbacks are in the form of total patient admission number. As the DES does not have the capability in capturing the feedback loop and SD does not have the capability of capturing the individual analysis, in order to make reliable and efficient decisions therefore, the policy makers need to look at both results generated by both models.

Table 1 presents the result from the DES model, which was executed for 2940 simulated hours (30 weeks $* 7$ days * 14 hours [0800 to 2200 everyday]). This result is generated after taking into consideration the patients total readmissions based on the assumption that each patient will continue their journey into care pathway from 0800 to 2200 hours, seven days per week. Data from the table shows that, with the current space available in intermediate care there are 20 spaces with maximum stay in intermediate care is 196 (14 hours * 14 days), average number patients waiting to enter the intermediate care is approx. 25 patients with average waiting time of 92.27 hours. Considering the result provided by the simulation, bed blocking may occur when a patient waiting to be admitted into intermediate care has to stay in healthcare until a space becomes available in intermediate care. As these are tangible factors that influence the total patient admission (total patient admission will influence stress level and non-patient recovery), any changes for intervention purposes, albeit with the same, will be inputted in the SD model to generate the feedback results. This is to ensure the synchronization of both models in term of their input, thus generating reliable result.

Table 1: DES Model Result

\begin{tabular}{|l|c|}
\hline \multicolumn{1}{|c|}{ Variables } & Current (intermediate care = 20) \\
\hline Total patient enter healthcare (patient) & 444.80 \\
\hline Total patient enter to intermediate care (patient) & 312.40 \\
\hline $\begin{array}{l}\text { Number of patient waiting for intermediate care } \\
\text { (patient) }\end{array}$ & 24.6 \\
\hline Average queuing time for intermediate care (hours) & 92.27 \\
\hline Minimum non zero waiting time (hours) & 0.97 \\
\hline Maximum queuing time (hours) & 240.60 \\
\hline Number of non-zero queuing time (patient) & 250.40 \\
\hline Standard deviation (hours) & 69.06 \\
\hline
\end{tabular}

\subsection{Result from the SD model}

The SD model provides result from the feedback loop based on intangible factors that are influenced by tangible factors (modeled by DES). Such results are in the form of total patient discharge from healthcare into intermediate care and total patient readmission. These are the results generated from intangible factors, i.e. staffs' level of stress and level of patient non-recovery, due to the total patient admission. The graphs shown in Figures 6 and 7 depict the level of stress and patient discharge rate respectively. dmnl (dimensionless) is the unit for patient/space. The higher the value of $d m n l$ (more than 1), the higher the rates of patient discharge. To maintain the connection and communication in both models, should the de- 
cision makers need to implement any intervention (e.g. added resources for reducing the total patient waiting and time waiting as well as to reduce the patient readmission), the intervention should be done in both models. This will also ensure that the results generated from both the DES and SD are reliable and can be used as a decision support tool. However, there are some conditions where this rule could not be applied. Some decision makers tend to reduce the patient readmission by educating their staff, as this tends to reduce the number of patient readmission. Should this happen, the rule of introducing intervention to both models can be ignored since DES is not capable of capturing intangible factors. This rule can also be ignored if the intervention is done to tangible factors and does not influence intangible factors.

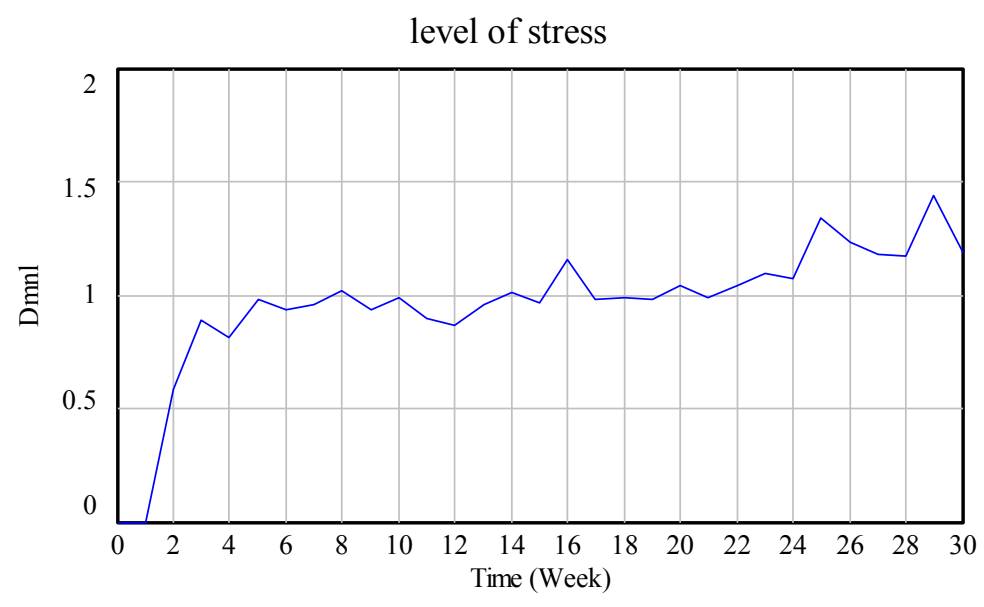

level of stress : Current

Figure 6: Level of Stress

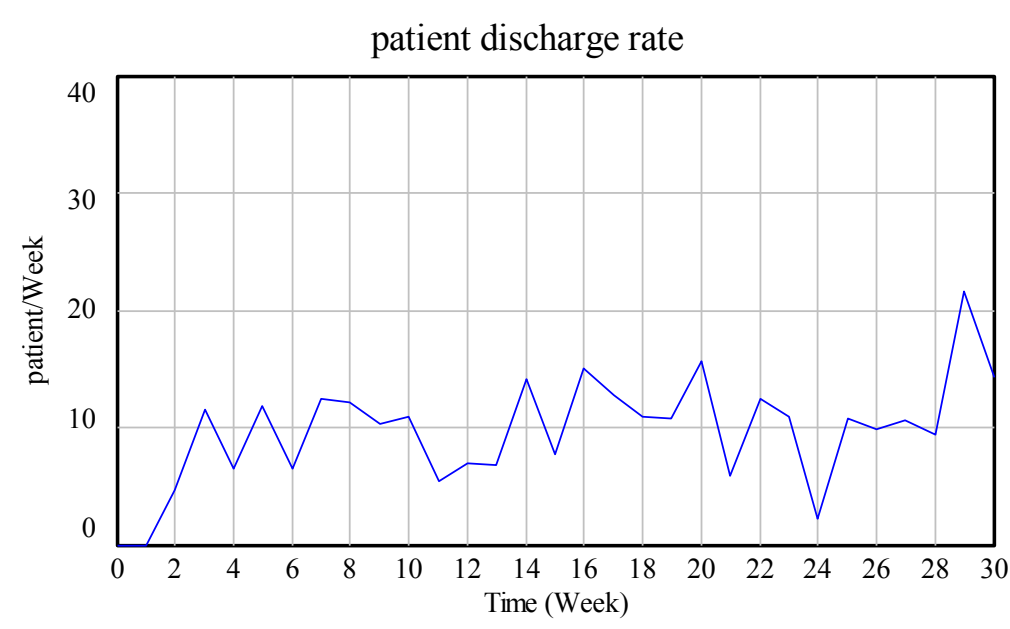

patient discharge rate : Current

Figure 7: Patient Discharge Rate

\section{CONCLUSION}

An important element in developing hybrid SD-DES models is to ensure that the underlying constituent models are balanced in terms of their respective inputs and outputs. Taking an example from the IC model, if the DES model has patients that have been discharged, then the flow for patient discharge should also be included in the SD model. This will ensure that decisions based on the output from both the SD and the DES models will be reliable and consistent. The need for developing system models using 
hybrid SD-DES techniques will depend on several factors, such as, its impacts on the current systems, the need for individuality analysis, and whether the system has a feedback element.

The work presented in this paper is of an experimental nature. Presently the results are based on a hybrid SD-DES model wherein variable values are transferred amongst the SD and the DES components. For future work we are planning to run further experiments on more complex situations; this will ensure realistic assessments of this approach. Furthermore, we aim to automate the process of transferring variable values between models in order to speed up the overall simulation execution process, this in addition to using other techniques such as Agent-Based Simulation. It must be noted that this proposed approach is not a substitute to existing technologies, rather provides a structured framework for modeling IC environments without starting from scratch.

\section{REFERENCES}

AbouRizk, S. M., and R. J. Wales. 1997. “Combined Discrete-Event/Continuous Simulation for Project Planning." Journal of Construction Engineering and Management 123(1):11-23.

Brailsford, S. C. 2008. "System Dynamics: What's In It for Healthcare Simulation Modellers." In Proceeding of the 2008 Winter Simulation Conference, Edited by S. J. Mason, R. R. Hill, L. Monch, O. Rose, T. Jefferson, and J. W. Fowler, 1478 - 1483. Piscataway, New Jersey: Institute of Electrical and Electronics Engineers, Inc.

Brailsford, S. C., P. R. Harper, B. Patel, and M. Pitt. 2009. "An Analysis of the Academic Literature on Simulation and Modelling in Healthcare." Journal of Simulation 3(3):130-140.

Brailsford S. C., and N. Hilton. 2001. "A Comparison of Discrete-Event Simulation and System Dynamics for Modelling Healthcare Systems." In Proceedings of the Operational Research Applied to Health Services (ORAHS 2001), Edited by J. Riley, 18-39.

Brailsford, S. C., W. J. Gutjahr, M. S. Rauner, and W. Zeppelzauer. 2007. "Combined Discrete-Event Simulation and Ant Colony Optimization Approach for Selecting Optimal Screening Policies for Diabetic Retinopathy." Computational Management Science 4(1):59-83.

British Geriatrics Society. 2008. Intermediate Care - Guidance to Commissioners and Providers of Health and Social Care. www.bgs.org.uk/index.php?option $=$ com_content\&view $=$ article\&id $=363$. Accessed May 28, 2012.

Bryan K., H. Gage, and K. Gilbert. 2006. "Delayed Transfers of Older People from Hospital: Causes and Policy Implementations." Journal of health policy 76(2):194-201.

Campbell, H., J. Karnon, and R. Dowie. 2001. "Cost Analysis of a Hospital-at-home Initiative Using Discrete Event Simulation." Journal of Health and Service Research Policy 6(1):14 - 22.

Chahal, K., and T. Eldabi. 2008. "Applicability of Hybrid Simulation to Different Modes of Governance in UK Healthcare." In Proceeding of the 2008 Winter Simulation Conference, Edited by S. J. Mason, R. R. Hill, L. Monch, O. Rose, T. Jefferson, and J. W. Fowler, 1469 - 1476. Piscataway, New Jersey: Institute of Electrical and Electronics Engineers, Inc.

Chahal K., T. Eldabi, and A. Mandal. 2009. "Understanding the Impact of Whiteboard on A\&E Department Using Hybrid Simulation." In Proceeding of $27^{\text {th }}$ International Conference of the System Dynamics Society, Albuquerque, New Mexico, USA. System Dynamics Society.

Desai M. S., M. L. Penn, S. C. Brailsford S., and M. Chipulu. 2008. "Modelling of Hampshire Adult Services - Gearing-up for Future Demands." Journal of Health Care Management Sciences 11(2):167176.

Elf, M., and M. Putilova. 2005. "The Care Planning Process - A Case for System Dynamics." In Proceedings of the $23^{\text {rd }}$ International Conference of the System Dynamic Society, paper 289. System Dynamics Society.

Fakhimi, M., and N. Mustafee. 2012. "Applications of Operations Research within the UK Healthcare Context". In Proceedings of the 2012 OR Society Simulation Workshop (SW12), Edited by B.Tjahjono, C. Heavey, S. Onggo, and D-J. van der Zee, 66-82. UK OR Society. 
Glendinning, C., B. Hudson, and R. Means. 2005. "Under Strain? Exploring the Troubled Relationship between Health and Social Care." Journal of public money and management, 25(4):245-251.

Helal, M., L. Rabelo, J. Sepúlveda, J. and A. Jones. 2007. "A Methodology for Integrating and Synchronizing the System Dynamics and Discrete Event Simulation Paradigms." In Proceedings of the 25th International Conference of the System Dynamics Society. System Dynamics Society.

Jun, J. B., S. H. Jacobson, and J. R. Swisher. 1999. "Application of Discrete-event Simulation in Healthcare Clinics: A Survey." Journal of the Operational Research Society 50(2):109-123.

Katsaliaki K., S. Brailsford, D. Browning, and P. Knight. 2005. "Mapping Care Pathways for the Elderly." Journal of Health Organization and Management 19(1):57-72.

Katsaliaki, K., and N. Mustafee. 2011. "Applications of Simulation Research within the Healthcare Context." Journal of the Operational Research Society 62(8):1431-1451.

Lane, D. C. 2000. "You Just Don't Understand: Modes of Failure and Success in the Discourse Between System Dynamics and Discrete-Event Simulation." Operational Research working papers, LSEOR 00.34. Department of Operational Research, London School of Economics and Political Science, London, UK.

Morecroft J. D. W., and S. Robinson 2005. "Explaining Puzzling Dynamics: Comparing the Use of System Dynamics and Discrete Event Simulation." In Proceedings of the $23^{\text {rd }}$ International Conference of the System Dynamics Society, Edited by J. D. Sterman, M. P. Repenning, R. S. Langer, J. I. Rowe, and J. M. Yarni. Boston, MA: System Dynamic Society.

Mur-Veeman, I., van Raak, A., and A. Paulus. 2008. "Comparing Integrated Care Policy in Europe: Does Policy Matter?" Journal of health policy, 85(2):172-183.

Mustafee, N., S. J. E. Taylor, K. Katsaliaki, and S. Brailsford. 2009. "Facilitating the Analysis of a UK National Blood Service Supply Chain Using Distributed Simulation." SIMULATION: Transactions of the Society of Modelling and Simulation International 85(2):113-128.

Mustafee, N., and E. E. Bischoff. 2011. "A Multi-methodology Agent-based Approach for Container Loading." In Proceedings of the 2010 Winter Simulation Conference, Edited by B. Johansson, S. Jain, J. Montoya-Torres, J. Hugan, and E. Yücesan, 665-677. Piscataway, New Jersey: Institute of Electrical and Electronics Engineers, Inc.

Mustafee, N., F. Hughes, K. Katsaliaki, and M.D. Williams. 2011. "Mapping the Capacity and Demand for Specialist Haematology Outpatient Services". In Proceedings of the 2011 Operational Research Applied to Health Services (ORAHS 2011), Edited by P.Harper, V.Knight, I.Vieira and J. Williams, 176-186. UK: School of Mathematics, Cardiff University (ISBN 978-0-9569158-0-1).

Rabelo, L., H. Eskandari, T. Shaalan, and M. Helal. 2007. "Value Chain Analysis using Hybrid Simulation and AHP." International Journal of Production Economics 105(2):536-547.

Sachdeva, R., T. Williams, and J. Quigley. 2006. "Mixing methodologies to enhance the implementation of healthcare operational research." Journal of the Operational Research Society 58(2):159-167.

Sterman, J.D. (2000). Business dynamics: Systems thinking and modeling for a complex world. Boston, Mass.: Irwin/McGraw-Hill.

Sweetser, A. 1999. "A Comparison of System Dynamics and Discrete Event Simulation." In Proceedings of 17th International Conference of the System Dynamics Society and the 5th Australian and New Zealand Systems Conference, Edited by R. Y. Cavana, J. A. M. Vennix, E. A. J. A. Rouwette, M. Stevenson-Wright, and J. Candlish, Paper 78. Wellington, New Zealand: System Dynamics Society.

van Raak, A., A. Paulus, and I. Mur-Veeman. 2003. "Why Do Health and Social Care Providers Cooperate." Journal of health policy, 74(1):13-23.

Wolstenhome, E., D. McKelvie, G. Smith, and D. Monk. 2004. "Using System Dynamics in Modelling Health and Social Care Commissioning in the UK." In Proceedings of the $22^{\text {nd }}$ International Conference of the System Dynamic Society, Edited by M. Kennedy, G. W. Winch, R. S. Langer, J. I. Rowe, and J. M. Yanni. Paper 116. System Dynamics Society. 


\section{AUTHOR BIOGRAPHIES}

JAFRI ZULKEPLI is a lecturer in Decision Sciences Department in the School of Quantitative Sciences, Universiti Utara Malaysia. He has Bachelor of Information Technology, M.Sc. in Information Technology (Management) and obtained his Ph.D. from Brunel University, UK, recently. His research is into aspects of information technology, Decision Support System, modeling and hybrid simulation, specifically, Discrete Event Simulation and System Dynamics. His email address is zhjafri@uum.edu.my.

TILLAL ELDABI is a senior lecturer at Brunel University. He has B.Sc.in Econometrics and M.Sc. and $\mathrm{Ph} . \mathrm{D}$. in Simulation Modeling in Healthcare. His research is into aspects of healthcare modeling and simulation. He developed a number of models and bespoke packages to support health economists and clinicians to decide on best treatment programs. Dr Eldabi has published widely in the field of modeling in healthcare and edited a number of special issues in highly respected journals. Dr. Eldabi's email address is tillal.eldabi@brunel.ac.uk.

NAVONIL MUSTAFEE is a lecturer in Operations Management and Information Systems in the School of Business and Economics, Swansea University (UK). His research interests are in simulation methodologies, healthcare simulation, parallel and distributed simulation, technology adoption and diffusion, and grid computing. His e-mail address is n.mustafee@swansea.ac.uk and his web page is http://sites.google.com/site/navonilmustafee/. 